\title{
Design of CW THz Photonic Transmitter based on Low Pass-Filter and Bow-tie Wideband Antenna
}

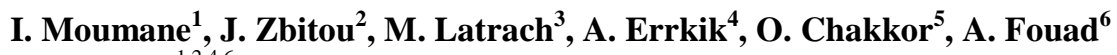 \\ ${ }^{1,2,4,6}$ LMEET, FSTS Hassan 1St University of Settat Morocco \\ ${ }^{3}$ Microwave group ESEO, Angers, France \\ ${ }^{5}$ RSAID ENSA of TETOUAN, Morocco
}

\section{Article Info}

Article history:

Received May 22, 2018

Revised Jul 30, 2018

Accepted Aug 6, 2018

Keyword:

Bow-tie antenna

$\mathrm{CW} \mathrm{THz}$ photonic transmitters

Low pass-filter

DC probe

GaAs substrate

\section{Corresponding Author:}

Ibtissame Moumane,

LMEET,

FSTS Hassan 1St University of Settat, Morocco.

Email: moumaneibti@gmail.com

\begin{abstract}
This paper presents the design of Continuous Wave Terahertz photonic transmitters which is composed from photodetector (PD) associated to a wideband antenna, low-pass filter (LPF) and DC Probe. Firstly, we have developed the bow-tie wideband antenna using an EM solver Momentum integrated in ADS "Advanced Design System". Then we had optimized a low-pass filter which is responsible of blocking the RF signal providing from the antenna to reach the DC probe. And finally, we have validated into simulation the $\mathrm{CW} \mathrm{THz}$ photonic transmitter. The three structures are based on multi-layers GaAs substrate, which is the most widely used for $\mathrm{THz}$ circuit design. The dimensions of the Whole circuit are $776.788 \times 303.39 \mu \mathrm{m}^{2}$.
\end{abstract}

Copyright (c) 2018 Institute of Advanced Engineering and Science. All rights reserved.

\section{INTRODUCTION}

We remark in the recent years the big interest giving to the terahertz technology. Before the $\mathrm{THz}$ domain was ignored due to a lot of difficulties related to the generation and detection. But with the development of the modern Femtosecond Lasers and High-Speed Photodetectors the situation was changed. A diversity of domains such as biomedical imaging, spectroscopy, Security and telecommunications are focus now theirs applications on $\mathrm{THz}$ waves which presented several advantages based on interactivity with the material where it spreads and fast absorption by the atmosphere [1].

To generate the THz waves many methods are proposed but the most used is the one that relays on the coplanar waveguide (CPW) photonic transmitters [2]. The CPW technology offers in fact several advantages due to its features, like low radiation, low dispersion, easy of shunts and series connections [3]. This paper presents a new study of a $\mathrm{THz}$ photonic transmitter composed from a photodetector associated to the broadband antenna inserts in series with a low pass filter and a DC Probe. This paper is divided on two parts, the first one is reserved to the theory of different components of the system and the second part presents the design of devices and discussion of simulation results.

\section{RESEARCH METHOD}

A PD is a sensor its role is to convert an optical power into an electrical current. To generate electron-hole-pairs, the photon energy provides from the light absorbed in a PD must be at last equal to the bandgap energy Eg of the absorber material [4].This available energy of one photon is sufficient to excite an electron from the valence band (v.b.) to the conduction band (c.b.). For this band-to-band transition, the upper 
wavelength limit for photon absorption is given by [4]:

$$
\lambda_{\mathrm{g}}[\mu \mathrm{m}]=\frac{1.24}{\mathrm{E}_{\mathrm{g}}[\mathrm{eV}]}
$$

A PD has different proprieties such as:

Sensitivity: The ability of the photodiode to transform light absorbed into an electrical current in other term the number of charge carrier pairs generated per incident photon [4].

$$
\eta_{\text {ext }}=\frac{I_{\mathrm{Pd}}}{\mathrm{q}} \cdot \frac{\mathrm{h} v}{\mathrm{P}_{\mathrm{opt}}}
$$

Responsivity: The external responsivity $\mathrm{R}$ is the ratio of photocurrent to the input optical power [4]:

$$
\mathrm{R}=\frac{I_{\mathrm{Pd}}}{\mathrm{P}_{\mathrm{opt}}}=\frac{\eta_{\mathrm{ext}} \lambda[\mu \mathrm{m}]}{1.24} A / W
$$

Where $\mathrm{R}$ depends on the state of polarization of the incoming light, the definition of the polarization dependent loss (PDL) is useful [4]:

$$
\mathrm{PDL}=10 \cdot \log \left(\frac{R_{\max }}{R_{\min }}\right) d B
$$

With Rmax and Rmin are the maximum and minimum responsivities for all states of polarization.

In order to evaluate the high-speed photodiode, the electrical $3 \mathrm{~dB}$ bandwidth is used, It specifies the frequency range from $\mathrm{DC}$ to the cut-off frequency $\mathrm{f}_{3 \mathrm{~dB}}$. The bandwidth of a lumped element photodiode, at which the device length is much shorter than the electrical signal wavelength, might be limited mainly by the RC-time constant and the carrier drift times in the depletion region. These bandwidth constraints lead to a $3 \mathrm{~dB}$ bandwidth which can be approximated by [4]:

$$
\mathrm{f}_{3 \mathrm{~dB}} \approx \sqrt{\frac{1}{\frac{1}{\mathrm{f}_{\mathrm{RC}}^{2}+\frac{1}{\mathrm{f}_{\mathrm{t}}^{2}}}}}
$$

With the RC limited bandwidth.

$$
\mathrm{f}_{\mathrm{RC}}=\frac{1}{2 \pi \mathrm{R}_{\mathrm{tot}} \mathrm{C}_{\mathrm{pd}}}
$$

And the carrier transit time limited bandwidth.

$$
\mathrm{f}_{\mathrm{t}}=\frac{3.5 \bar{\vartheta}}{2 \pi \mathrm{d}_{\mathrm{abs}}}
$$

$\mathrm{R}_{\text {tot }}$ stands for the photodiodes's total resistance including series and load resistances.

$\mathrm{C}_{\mathrm{pd}}$ is the junction capacitance.

$\bar{\vartheta}=\sqrt[4]{2\left(1 / v_{\mathrm{e}}^{4}+1 / \mathrm{v}_{\mathrm{h}}^{4}\right)^{-1}}$ is the averaged carrier drift velocity with the electron and hole velocities $V_{\mathrm{e}}$ and $\mathrm{V}_{\mathrm{h}}$.

$\mathrm{d}_{\mathrm{abs}}$ : The absorber thickness [4].

\subsection{Photodetector structures}

There is a different structure of high speed detectors which are shown in Figure 1:

In our case we used The Metal Semiconductor-Metal Traveling wave Photodetector (MSM-TPD) by reason of its high power-bandwidth and coplanar-waveguide fed slot owing to its easy connection with planar devices [5]. The PD based on GaAs substrate which characterized by a succession of layers as mentioned in the Figure $2[6]$ : 
(a)

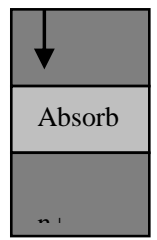

(b)

(c)

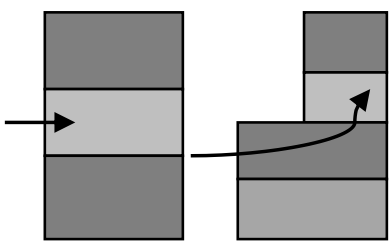

(e) (d)

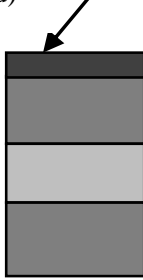

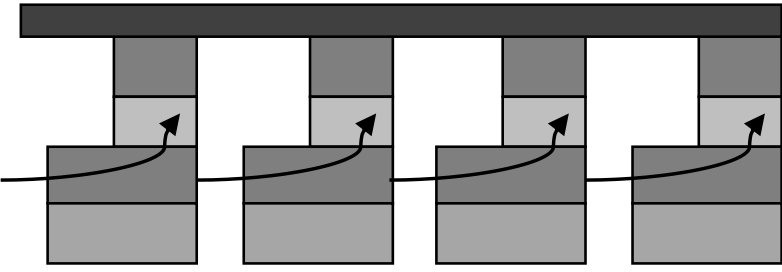

Figure 1. High speed photodiodes (a) vertically illuminated PD, ( b) waveguide PD, (c) waveguide-integrated PD, (d) TWPD with continuous absorption, (e) periodic waveguide-integrated TWPD [4].

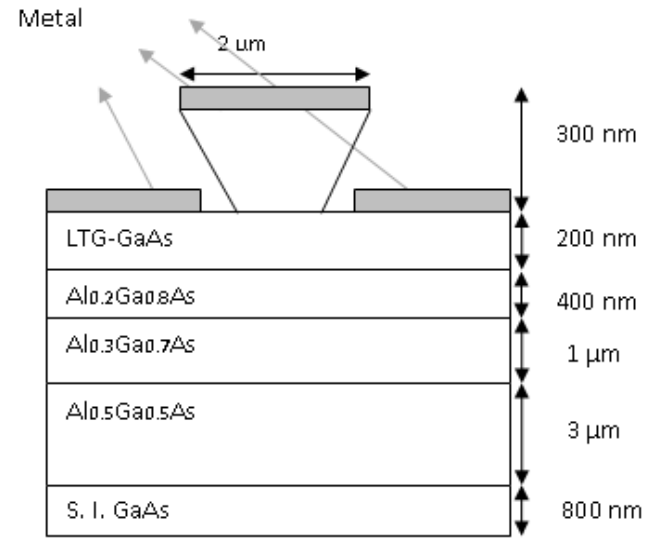

Figure 2. Structure of the photodetector based on GaAs substrate

\subsection{THz wideband antenna}

The antenna is one of the most important elements in the design of CPW photonic transmitter [7], [8]. Its role focus on the transmission of RF signals providing from PD.

In this work we choose the Bowtie design for the antenna which is a planar version of the well known biconical antenna [2].This antenna type gives a wideband frequency transmission in the THz domain. Figure 3 shows the proposed antenna structure.

To optimize the final antenna structure we have used Momentum integrated in ADS [9]. After many series of optimization by using many methods as Random and gradient, we have validated the structure depicted in Figure 3. The Table 1 presents different parameters of the optimized Bowtie antenna.

Figure 4 presents the reflection coefficient of the validated antenna with the matching input impedance below $-10 \mathrm{~dB}$ between $1 \mathrm{THz}$ and $1.25 \mathrm{THz}$. That makes it suitable for $\mathrm{THz} \mathrm{CW}$ photonic transmitters.

To study the radiation behavior of the antenna we have launched a 3D simulation at $1.22 \mathrm{THz}$ in ADS, we obtain the radiation diagram which shows that the antenna is bidirectional as shown In Figure 5. 


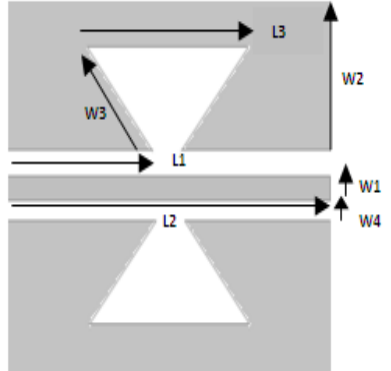

Figure 3.The Bowtie antenna structure

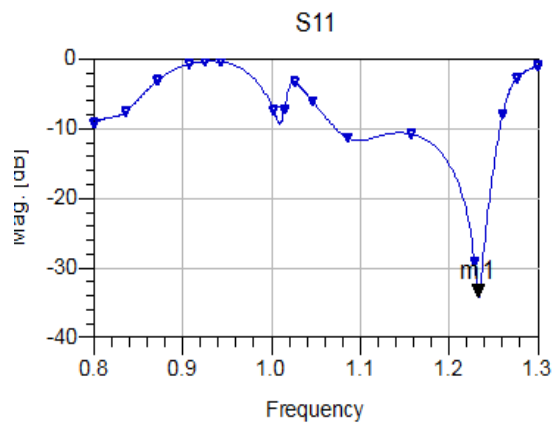

Figure 4. Reflection coefficient versus frequency
Table 1. Values of Design Parameters

\begin{tabular}{cc} 
Parameter & Value $(\mu \mathrm{m})$ \\
\hline L1 & 147.26 \\
L2 & 330 \\
L3 & 223.497 \\
W1 & 15 \\
W2 & 100,23 \\
W3 & 132,71 \\
W4 & 11.75 \\
\hline
\end{tabular}

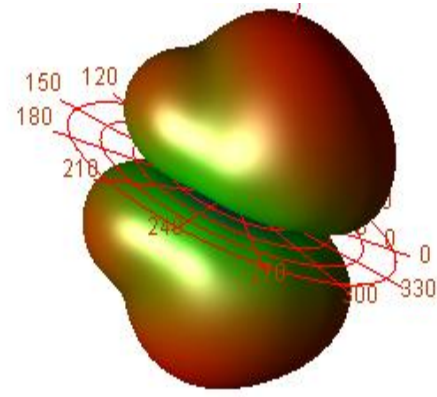

Figure 5. The radiation pattern at $1.22 \mathrm{THz}$

\subsection{Low-pass filter (LPF)}

The integration of the Low Pass Filter into the CW photonic transmitter system is mandatory. It works like an inductance which role reside in blocking the RF signal providing from PD and transmitting via antenna to reach the DC probe.

In this paper we choose a several periodic structure presenting in the study [10] with the aim to increase the rejection band and to obtain a low insertion loss in the pass-band along a CPW line as demonstrated in [11]. The proposed structure is composed from three units as shown in Figure 6 and dimensions are presented in Table 2.

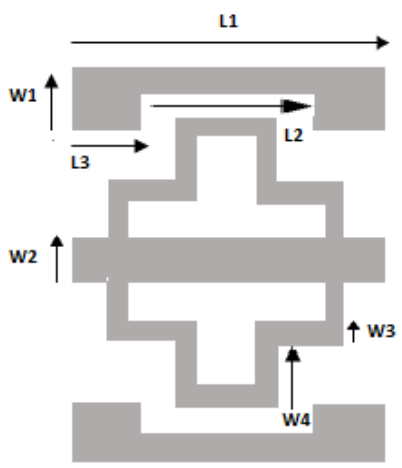

Table 2. Unit Cell of Periodic Structure CPW LowPass Filter Parametres

\begin{tabular}{cc}
\hline Parameter & Value $(\mu \mathrm{m})$ \\
\hline$L 1$ & 77.61 \\
L2 & 30.28 \\
L3 & 25.70 \\
$W 1$ & 20.77 \\
$W 2$ & 25 \\
$W 3$ & 5 \\
$W 4$ & 15.5 \\
\hline
\end{tabular}

Figure 6. Unit cell of periodic structure CPW lowpass Filter

The idea was to validate a $\mathrm{THz}$ low pass filter based on this cell by enlarging the rejected band. Therefore, we have validated into simulation after the optimization of this structure in Momentum by achieving a periodic low pass filter (LPF) based on the unit cell presents in Figure 7. This final filter is mounted on a multilayers GaAs substrate [6]. 
The Table 3 resumes the different dimensions of the proposed LPF.

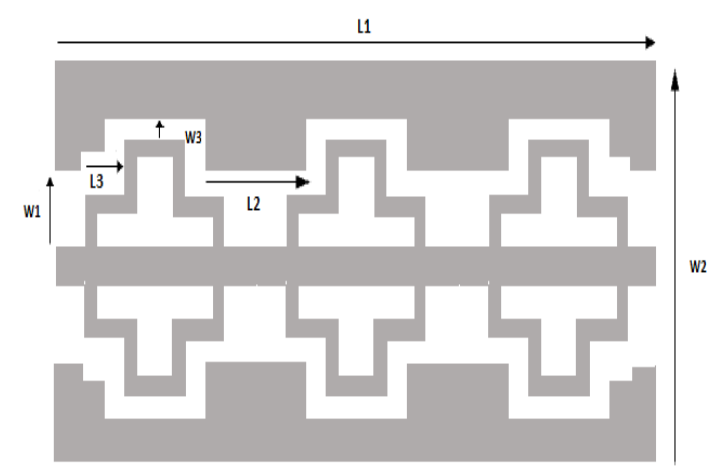

Table 3. Values of Filter Parameters

Figure 7. The layout of the periodic LPF THz structure

Figure 8 demonstrates that the achieved LPF presents a good low insertion loss, with a cutoff frequency of $0.67 \mathrm{THz}$ and a wide rejection band until $1.35 \mathrm{THz}$, which makes the proposed "LPF" able to work in $\mathrm{THz}$ domain and to be insert into a $\mathrm{THz}$ system of $\mathrm{CW}$ photonic transmitters [10].

To validate the function of the "LPF" structure we have launched a 3D simulation in ADS at 0.55 $\mathrm{THz}$ in the frequency passband and at $1 \mathrm{THz}$ in the rejection frequency band. As a result, we can see from Figure 9(a) that we have a flow of energy from port 1 to port 2 and for Figure 9(b) the energy is stoped to reach the port 2 .

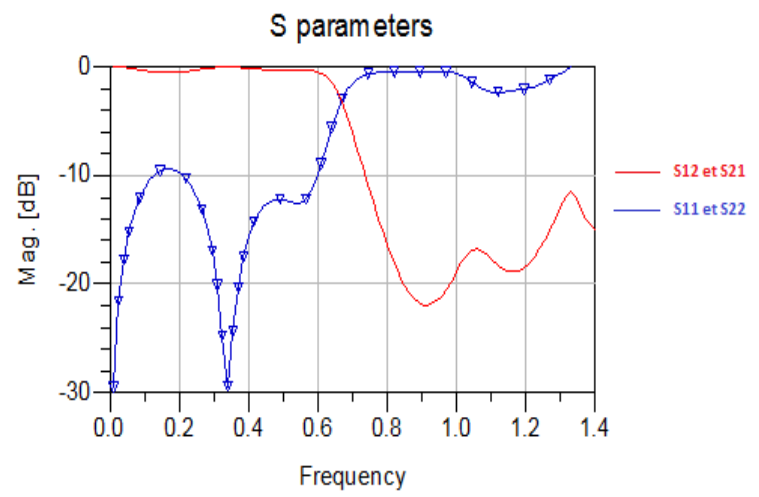

Figure 8. Simulation S-parameters results versus frequency

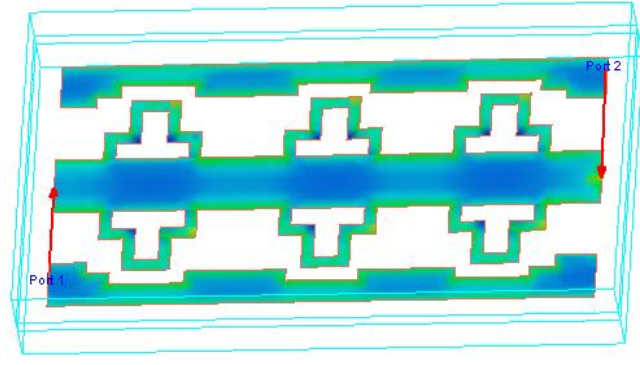

(a)

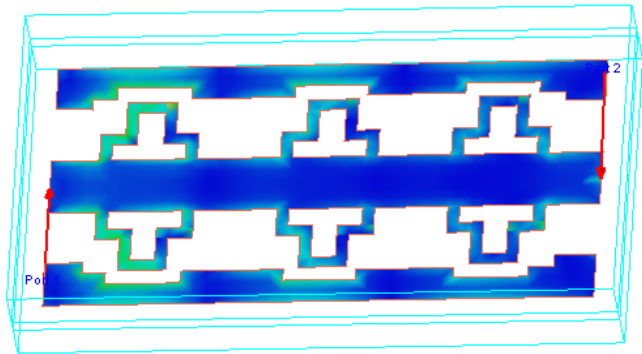

(b)

Figure 9. The current density @ (a) $0.55 \mathrm{THz}$ and (b) $1 \mathrm{THz}$ 


\subsection{CW Photonic transmitter simulation}

After the simulation of the antenna and the filter separately, we have connected the photodetector to the system composed from the antenna, the filter and DC probe responsible for the polarization. The global system named CW photonic transmitter for generation of THz signal is presented in Figure 10.

Table 5 shows the values of DC probe parameters.

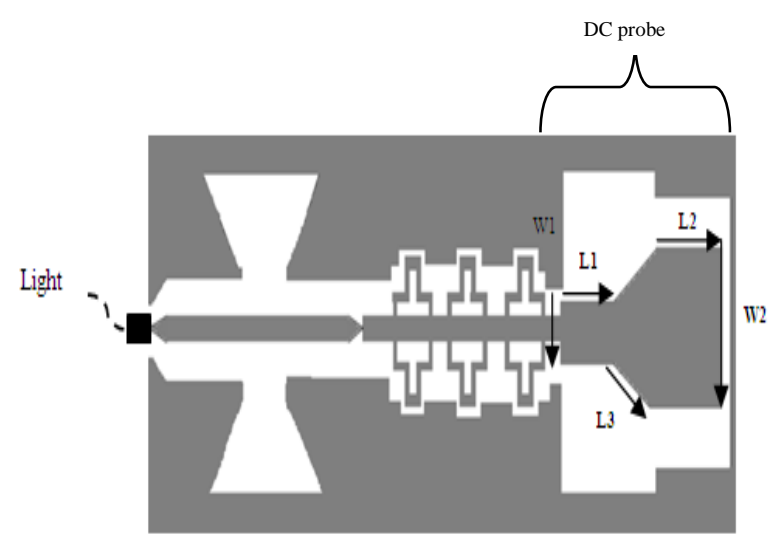

Table 5. Values of DC Probe Parameters

\begin{tabular}{cc} 
Parameter & Value $(\mu \mathrm{m})$ \\
\hline$L 1$ & 60.74 \\
$L 2$ & 62.91 \\
$L 3$ & 88.77 \\
$W 1$ & 36.88 \\
$W 2$ & 110.64 \\
\hline
\end{tabular}

Figure 10. The proposed CW photonic transmitter

As mentioned, we have associated the antenna, LPF with the DC probe having the optimized dimensions illustrated in Table 6. As shown in Figure 11, the simulation of the final $\mathrm{CW} \mathrm{THz}$ photonic transmitter permits to validate this circuit between $750 \mathrm{GHz}$ and $980 \mathrm{GHz}$.

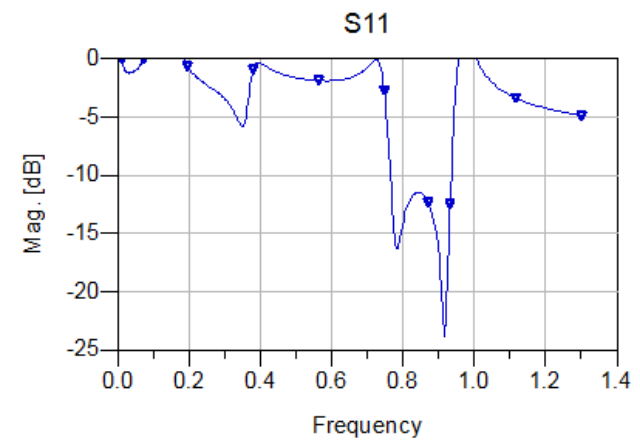

Figure 11. Simulation S-Parameters results versus frequency

After the validation of this $\mathrm{THz}$ photonic transmitter into simulation we have done a comparison between the proposed $\mathrm{THz}$ antenna which is the key of the $\mathrm{THz}$ source and with another $\mathrm{THz}$ antennas validated in literature. The table below presents the comparaison of the performances (dimensions, frequency bandwidth) between the proposed antenna and two others structures:

As shown in table 6, the proposed antenna presents good performances in term of bandwidth with an acceptable miniature length.

Table 6. Comparaison of the Antenna structures

\begin{tabular}{ccc}
\hline Antenna Structure & Length & Frequency Bandwidth \\
\hline Proposed Antenna & $330 \mu \mathrm{m}$ & [1THz,1.25THz] \\
Antenna [12] & $200 \mu \mathrm{m}$ & Narrow band at $645 \mathrm{Ghz}$ \\
Antenna [13] & $1040 \mu \mathrm{m}$ & Narrow band at $850 \mathrm{Ghz}$ \\
\hline
\end{tabular}




\section{CONCLUSION}

This paper presented a CW photonic transmitter used for generation of $\mathrm{THz}$ waves. To obtain the final target we have follow four main steps. Firstly, we have chosen the photodector, in our case we have used MSM-TPD. Secondly we have optimized the Bowtie antenna to be able to transmit and receive $\mathrm{THz}$ signal. In the third step, we have validated the "LPF" as an inductance to block the RF signal from reaching the DC probe. And finally, we have generated and optimized the whole $\mathrm{CW} \mathrm{THz} \mathrm{photonic} \mathrm{transmitter} \mathrm{by}$ using an electromagnetic solver Momentum integrated in ADS. The final circuit is mounted on a multilayers GaAs substrate and having an area around $776.788 \times 303,39 \mu^{2}$.

\section{ACKNOWLEDGEMENTS}

We thank Mr. Mohamed LATRACH Professor in ESEO, Engineering Institute in Angers, France, for allowing us to use all the equipments and EM solvers available in his laboratory.

\section{REFERENCES}

[1] Bertrand Gauvreau, Ondes terahertz, Ecole Polytechnique de Montréal, 2500 chemin de Polytechnique, Montréal, QC H3T 1J4, Canada.

[2] J. Zbitou, et al., "Bow-tie Wideband Antenna Design for CW THz Photonic Transmitters", Progress in Electromagnetics Research Symposium, Cambridge, USA, July 2-6, 2008.

[3] S. Khireddine, et al., "Flat Group Delay Low Pass Filters Using Two CPW Topologies", * IETR, CNRS UMR 6164, INSA Rennes, France, *DAlightCom, Lannion, France.

[4] Vorgelegt von Diplom-Physiker Andreas Beling aus Bonn, Periodic Travelling Wave Photodetectors with Serial and Parallel Optical Feed Based on InP.

[5] Shi, J.-W., et al., "Metal-Semiconductor-Metal traveling-wave Photodetectors", IEEE Photonics Technology Letters, vol. 13, no. 6, pp. 623-625, June 2001.

[6] Chi-KuangSun, THz Optoelectronics, Ultra Fast Optics Laboratory (UFO) Graduate Institute of Electro-Optical Engineering and Department of Electrical Engineering National Taiwan University Taipei, TAIWAN.

[7] Rachid Dakir, et al., "A New Compact and Miniaturized GCPW-fed Slotted Rectangular antenna for Wideband UHF FIRD Applications”, IJECE International Journal of Electrical and Computer Engineering, vol. 7, no. 2, April 2017.

[8] Abdelhadi Ennajih, et al., "A New Dual Band Printed Metamaterial Antenna for RFID Reader Applications”, IJECE International Journal of Electrical and Computer Engineering, vol. 7, no. 6, December 2017.

[9] Advanced Design System : https://www.keysight.com/.

[10] I. Moumane, et al., "A New Topology of a Low-Pass Periodic Filter for THz Photonic Transmitter System", ICCWCS'16 FST de Settat.

[11] Fouad Aytouna, et al., "A Novel Cpw Low Cost Lowpass Filter Integrating Periodic Structures", IJECE International Journal of Electrical and Computer Engineering, vol. 6, no. 3, June 2016.

[12] Ming-Chun Tien, Hsu-Hao Chang, Ja-Yu Lu, Li-Jin Chen, Shih-Yuan Chen, Ruey-Beei Wu, Senior Member, IEEE,Wei-Sheng Liu, Jen-Inn Chyi, Senior Member, IEEE, and Chi-Kuang Sun, Senior Member, IEEE, "Device Saturation Behavior of Submillimeter-Wave Membrane Photonic Transmitters".

[13] Yi-Chun Chen, et al., "Design of Rampart Slot Array Antenna in Integrated 850GHz Photonic Transmitter", Department of Electrical Engineering and Graduate Institute of Communication Engineering, Graduate Institute of Electro-Optical Engineering.

\section{BIOGRAPHIES OF AUTHORS}

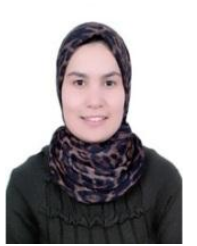

Ibtissame Moumane was born in Morocco in 1989, She received the degree of engineering in telecommunication and network from National School of Applied Sciences of Khouribga, Morocco in 2012. Currently, She is preparing the Ph.D. in University of Hassan $1^{\text {st }}$ in Settat. Her interests include $\mathrm{THz}$ communication, antennas, low-pass filters, photodetectors and CW Photonic Transmitter.

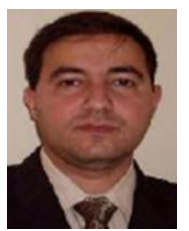

Jamal Zbitou was born in Fes, Morocco, in June 1976. He received the Ph.D. degree in electronics from Polytech of Nantes, the University of Nantes, Nantes, France, in 2005. He is currently an associate Professor of Electronics in FST University Hassan 1st, Settat, Morocco. He is involved in the design of hybrid, monolithic active and passive microwave electronic circuits. 


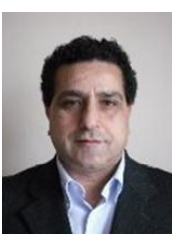

Mohamed Latrach was born in Douar Ksiba, Sless, Morocco, in 1958. He received the Ph.D. degree in electronics from the University of Limoges, Limoges, France, in 1990. He is currently a Professor of microwave engineering with the Ecole Suprieure d'Electronique de l'Ouest (ESEO), Angers, France, where his research involves RF and microwaves. His field of interest is the design of hybrid, monolithic active, and passive microwave circuits, metamaterials, LH materials, antennas and their applications in wireless Communications.

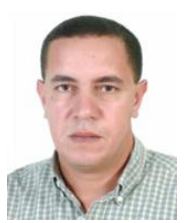

Ahmed Errkik was born in July 1960 in Morocco. He received the Ph.D. degree in physics from the University of Technology Compiegne (UTC), Compiegne, France. He is currently an associate Professor of physics in FST University Hassan 1st, Settat, Morocco. He is involved in the design of hybrid, monolithic active and passive microwave electronic circuits. 